\title{
MicroRNA-27b reverses docetaxel resistance of non-small cell lung carcinoma cells via targeting epithelial growth factor receptor
}

\author{
SHI CHEN ${ }^{1,2}$, QIAN WANG ${ }^{2}$, XIAN-MEI ZHOU ${ }^{2}$, JI-PING ZHU $^{2},{\text { TIAN } \text { LI }^{3} \text { and MAO HUANG }}^{1}$ \\ ${ }^{1}$ Department of Respiratory Disease, First Affiliated Hospital of Nanjing Medical University; \\ ${ }^{2}$ Department of Respiratory Medicine, Affiliated Jiangsu Province Hospital of Traditional Chinese Medicine, \\ Nanjing University of Traditional Chinese Medicine; ${ }^{3}$ Department of Respiratory Medicine, \\ Nanjing Chest Hospital, Nanjing, Jiangsu 210029, P.R. China
}

Received April 11, 2015; Accepted January 7, 2016

DOI: $10.3892 / \mathrm{mmr} .2016 .5332$

\begin{abstract}
MicroRNA (miR)-27b has been reported to participate in regulating the activity of non-small cell lung carcinoma (NSCLC) cells. Additionally, when downregulated in NSCLC it promotes resistance to docetaxel; however, the underlying molecular mechanism remains largely unknown. Using reverse transcription-quantitative polymerase chain reaction, the present study determined that the expression of miR-27b was significantly reduced in NSCLC cells that were resistant to docetaxel. In addition, epidermal growth factor receptor (EGFR) was identified as a possible target of miR-27b by searching the online miRNA database, TargetScan. A luciferase assay further validated EGFR as an effective target gene of miR-27b. In addition, it was determined that in tumor tissue samples resistant to docetaxel miR-27b was significantly downregulated, whilst EGFR was significantly upregulated. miR-27b negatively regulated the expression of EGFR. This was evident as the transfection of miR-27b mimics led to downregulation of the expression levels of EGFR, whilst miR-27b inhibitors upregulated the expression levels of EGFR. Furthermore, it was demonstrated that the transfection of miR-27b mimics significantly suppressed the apoptosis and promote the viability of A549 human lung carcinoma cells. In line with this, the introduction of miR-27b inhibitors significantly induced apoptosis and inhibited the proliferation of A549 cells. These results indicate that miR-27b may promote NSCLC cell viability and enhance resistance to docetaxel treatment through direct inhibition of EGFR expression. Additionally, miR-27b may become a promising molecular target for improving the effectiveness of chemotherapy with docetaxel.
\end{abstract}

Correspondence to: Dr Mao Huang, Department of Respiratory Disease, First Affiliated Hospital of Nanjing Medical University, 300 Guangzhou Road, Nanjing, Jiangsu 210029, P.R. China

E-mail: huang_mao11@163.com

Key words: microRNA-27b, non-small cell lung carcinoma, epithelial growth factor receptor, docetaxel, resistance

\section{Introduction}

Non-small cell lung carcinoma (NSCLC) is the most common type of lung cancer and is often fatal (1). NSCLC can be divided into different subtypes including the common squamous cell carcinoma and adenocarcinoma and the less common, large cell lung carcinoma. NSCLC accounts for $70-80 \%$ of lung cancer diagnoses, and the disease has often progressed to an advanced stage by the time it is detected (1). Although various treatments have been investigated, systemic chemotherapy has been indicated to improve the quality of life and the survival of patients with advanced NSCLC (1).

As innovative microtubule stabilizing agents, taxanes, including paclitaxel and docetaxel, have been clinically used for the treatment of NSCLC (2). Clinical efficacy of these agents varies from patient to patient, with a number of individuals exhibiting resistance to docetaxel. Resistance to chemotherapy remains a challenge in improving the quality of life and prolonging the survival of the patients with advanced stage NSCLC. Therefore, it is important to explore the underlying molecular mechanisms.

MicroRNAs (miRNAs) are a class of non-coding RNAs (18-25 nucleotides in length), that may bind to the 'seed sequences' in the 3'-untranslated region (3'-UTR) of mRNAs and thus trigger mRNA degradation or inhibit protein translation. In a previous study, miRNAs were shown to have a negative impact on cell survival, proliferation and motility by regulating the expression of their targets (3). In addition, miRNAs have been reported to act as oncogenes or tumor inhibitors. It has also been indicated that disruption of miRNA functioning is correlated with human malignant tumors (4). Furthermore, the deregulation of certain miRNAs, including miR-7, miR-182, miR-192 and miR-17, was associated with resistance and sensitivity to chemotherapy in NSCLC cells (5-8).

In a previous study, miR-27b was shown to be significantly downregulated in NSCLC tumor tissue that was resistant to docetaxel, as compared with tumor tissues that were sensitive to the treatment (9). The present study aimed to investigate the expression levels of miR-27b in samples collected from patients with NSCLC who were resistant or sensitive to docetaxel. In addition, the online miRNA database TargetScan was searched in order to identify the target genes of miR-27b, 
and a luciferase assay was conducted in order to confirm these predictions. Furthermore, the potential mechanisms underlying the involvement of miR-27b in the apoptosis of A549 human lung carcinoma cells, as well as their chemoresistance, were investigated.

\section{Materials and methods}

Tissue specimen collection. A total of 54 snap-frozen histologically confirmed NSCLC tissue samples were collected and evaluated for miRNA and gene expression. All of the samples were collected from the First Affiliated Hospital of Nanjing Medical University, and this study protocol was approved by the Institutional Review Boards at the First Affiliated Hospital of Nanjing Medical University. None of the patients had received any chemotherapy or radiotherapy prior to the surgery. Following surgical intervention, patients received at least 2-4 cycles of the docetaxel-based chemotherapy as the first-line adjuvant therapy. Patients who experienced recurrent or progressive disease during docetaxel-based chemotherapy or within 12 months post-completion of first line therapy were considered as docetaxel-resistant $(n=26)$. Patients with no recurrence or with recurrences beyond 12 months were defined as docetaxel-sensitive $(n=28)$. Informed consent was obtained from each patient. Following surgical removal, the tissue samples were stored in liquid nitrogen for future use.

Target prediction. The online miRNA database, TargetScan (www.targetscan.org), was used to predict the target genes of miR-27b, and the similarity of potential 'seed sequences' among various species was compared and presented.

Cell lines and cell culture. A549 cells were obtained from the Shanghai Institute of Biochemistry and Cell Biology (Shanghai, China). All cells were incubated in RPMI 1640 (Gibco; Thermo Fisher Scientific, Inc., Waltham, MA, USA) containing $100 \mathrm{U} / \mathrm{ml}$ penicillin, $10 \%$ fetal bovine serum and $100 \mathrm{~g} / \mathrm{ml}$ streptomycin (Invitrogen; Thermo Fisher Scientific, Inc.) in humidified air at $37^{\circ} \mathrm{C}$ at an atmosphere of $5 \% \mathrm{CO}_{2}$.

Transfection. Following culturing the cells to $70 \%$ confluence, they were transfected using Lipofectamine 2000 (Thermo Fisher Scientific, Inc.), according to the manufacturer's protocol. Briefly, miR-27b mimics/inhibitor and EGFR small interfering (si)RNA (all from Shanghai GenePharma Co., Ltd., Shanghai, China) were diluted in serum-free medium (1:50; Thermo Fisher Scientific, Inc.), which was also used to dilute Lipofectamine 2000 . Following incubation of the cells at $37^{\circ} \mathrm{C}$ in $5 \% \mathrm{CO}_{2}$ for $6 \mathrm{~h}$, the medium in each well was replaced by the normal serum-containing medium and cultured for $24 \mathrm{~h}$ prior to the assays being performed.

Western blot analysis of target genes. Cells or tissue samples were suspended in $50 \mu 1$ lysis buffer [ $1 \mathrm{mM}$ ethylene diamine tetraacetic acid, $\mathrm{pH} 8.0 ; 5 \mathrm{mM}$ dithiothreitol; $2 \%$ sodium dodecyl sulfate (SDS); and $50 \mathrm{mM}$ Tris- $\mathrm{HCl}, \mathrm{pH}$ 8.0] for $30 \mathrm{~min}$ in ice and then harvested. The resulting lysates were centrifuged at $4,000 \mathrm{x}$ g for $10 \mathrm{~min}$, and the supernatant was removed. A 10\% SDS-polyacrylamide gel electrophoresis membrane (Invitrogen; Thermo Fisher Scientific, Inc.) was used to resolve the proteins, which were electroblotted onto nitrocellulose membrane (Merck Millipore, Darmstadt, Germany). Subsequently, 5\% skimmed milk was used to block the blots. The membranes were then incubated with mouse anti-EGFR antibody $(1: 1,000 ;$ sc-120) and $\beta$-actin antibody (1:10,000; sc-130656; both Santa Cruz Biotechnology, Inc., Santa Cruz, CA, USA) at room temperature for $2 \mathrm{~h}$, and then incubated with horseradish peroxidase-conjugated anti-goat and mouse secondary antibodies (Sigma-Aldrich). The bands were observed with an Enhanced Chemiluminescence kit (Invitrogen; Thermo Fisher Scientific, Inc.). The relative density of the bands was quantified using ImageJ software 1.41 (National Institutes of Health, Bethesda, MA, USA), and normalized to the internal control, $\beta$-actin.

Reverse transcription-quantitative polymerase chain reaction $(R T-q P C R)$. Total RNA was extracted from the A549 cells using TRIzol reagent (Invitrogen; Thermo Fisher Scientific, Inc.). RNA samples $(10 \mu \mathrm{g})$ were reverse-transcribed into cDNA using the SuperScript III Reverse Transcriptase or miRNA-specific primers and the TaqMan MicroRNA Reverse Transcription kit (all: Thermo Fisher Scientific, Inc.), according to the manufacturer's protocol. PCR was conducted using $1 \mu \mathrm{l}$ cDNA, TaqMan MicroRNA Assays, TaqMan Universal PCR Master Mix, No AmpErase UNG and primers (Applied Biosystems; Thermo Fisher Scientific, Inc.), on an ABI 7500 Real-Time PCR system (Applied Biosystems; Thermo Fisher Scientific, Inc.). The PCR cycling conditions were as follows: $95^{\circ} \mathrm{C}$ for $30 \mathrm{sec}$, followed by 36 cycles at $95^{\circ} \mathrm{C}$ for $5 \mathrm{sec}$ and $60^{\circ} \mathrm{C}$ for $40 \mathrm{sec}$. The primer sequences were as follows: miR-27b forward, 5'-GGGGTTCAAGTAATTCAGG-3' and reverse, 5'-CAG TGCGTGTCGTGGA-3'; EGFR forward, 5'-GTCAGCTAG CGCGTATGCTAAT-3' and reverse, 5'-GCGATCGTCGTA TATCTAGTCAG-3'; and $\beta$-actin forward, 5'-GACCTCTAT GCCAACACAGT-3' and reverse, 5'-AGTACTTGCGCTCAG GAGG-3' (Sangon Biotech, Co., Ltd., Shanghai, China). The PCR products were separated by $1 \%$ agarose gel electrophoresis (Sigma-Aldich, St. Louis, MO, USA). Each RT-qPCR assay was repeated at least three times. The mRNA expression levels of the miRNA were normalized to U6 snRNA, whereas those of the target genes were normalized to $\beta$-actin. Relative expression levels were calculated using the $2^{-\Delta \Delta \mathrm{Cq}}$ method (10).

Dual luciferase reporter assay. The full length wild-type EGFR 3'-UTR was amplified by PCR, and inserted into a psiCHECK 2 vector (Promega Corporation, Madison, WI, USA), and the mutant type 3'-UTR of EGFR was generated using site-directed Mutagenesis kit (Agilent Technologies, Inc., Santa Clara, CA, USA). The psiCHECK2-wildtype-EGFR-3'-UTR or psiCHECK2-mutant1 or 2 EGFR-3'-UTR vectors were used to transfect the A549 cells, in the presence or absence of $100 \mathrm{nM}$ miR-27b mimics. Following transfection, the A549 cells were cultured at $37^{\circ} \mathrm{C}$ at an atmosphere of $5 \% \mathrm{CO}_{2}$ for $48 \mathrm{~h}$. The luciferase activity was then determined using an LD400 luminometer (Beckman Coulter, Inc., Brea, CA USA). The activity of firefly luciferase was standardized to the activity of Renilla.

3-(4,5-Dimethylthiazol-2-yl)-2,5-diphenyltetrazolium bromide (MTT) assay. The viability of the A549 cells was examined $48 \mathrm{~h}$ 


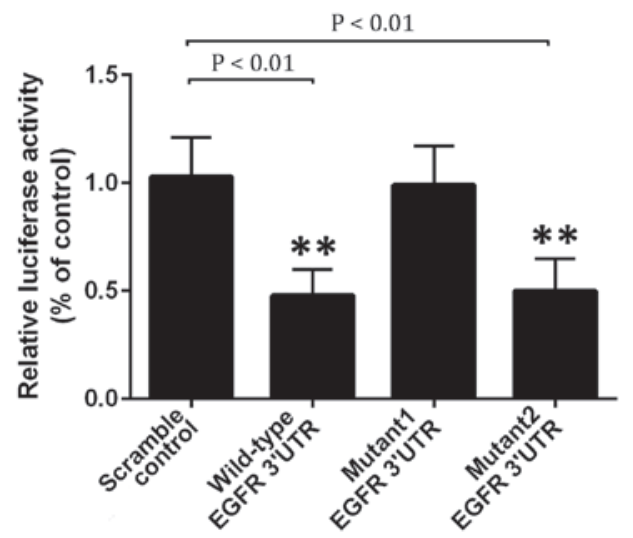

Figure 1. Luciferase activity was notably reduced in A549 cells co-transfected with the wild type or mutant 2 of EGFR 3'-UTR and miR-27b mimics, suggesting that miR-27b may directly bind to the 200-207 bp region in the 3'-UTR of EGFR mRNA in A549 cells ( ${ }^{* *} \mathrm{P}<0.01$ vs. control). EGFR, epithelial growth factor receptor; 3'-UTR, 3'-untranslated region.

following transfection. A549 cells $\left(5 \times 10^{3}\right.$ cells/well $)$ were seeded in a 96 -well plate and were incubated to $70-80 \%$ confluence. Subsequently, $10 \mu \mathrm{l}$ of $5 \mathrm{mg} / \mathrm{ml}$ MTT (Sigma-Aldrich, Shanghai, China) was added to all wells and cultured at $37^{\circ} \mathrm{C}$ for a further $4 \mathrm{~h}$. Following the removal of the medium, $150 \mu \mathrm{l}$ dimethyl sulfoxide (Sigma-Aldrich, Shanghai, China) were added to dissolve the MTT formazan. The optical density was determined at $490 \mathrm{~nm}$ using a spectrometer (ND-1000 Fluorospectrometer; Thermo Fisher Scientific, Inc.).

Apoptosis assay using flow cytometry. The A549 cells transfected with miR-27b mimics/inhibitor or EGFR siRNA were stained with Annexin V-fluorescein isothiocyanate and propidium iodide (PI) (Roche Diagnostics $\mathrm{GmbH}$, Mannheim, Germany). Following collection and washing with phosphate-buffered saline, the cells $\left(1 \times 10^{4}\right)$ were cultured with Annexin V and PI at room temperature for $20 \mathrm{~min}$. Flow cytometry (LSR II; BD Biosciences, San Jose, CA, USA) was used to analyze the A549 cells with a cell sorter activated by fluorescence (BD Biosciences).

Statistical analysis. All data are expressed as the mean \pm standard deviation, and the difference between the groups was evaluated using an independent t-test or one-way analysis of variance. $\mathrm{P}<0.05$ was considered to indicate a statistically significant difference. Each experiment was performed three times. Statistical analysis was performed using SPSS 19.0 (SPSS Inc., Chicago, IL, USA).

\section{Results}

EGFR is a direct target of $m i R-27 b$. miR-27b was significantly downregulated in docetaxel-resistant NSCLC, which may be involved in the molecular mechanism underlying the resistance to docetaxel (9). When computational analysis was performed in order to identify the potential target gene of miR-27b, the results of bioinformatic analysis predicted a regulatory association between miR-27b and EGFR (data not shown). In addition, the putative 'seed sequences' for miR-27b in the 3'-UTR of EGFR were evolutionarily conserved. To validate
A

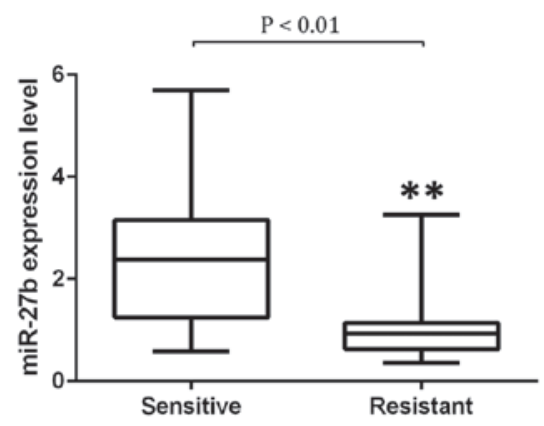

B

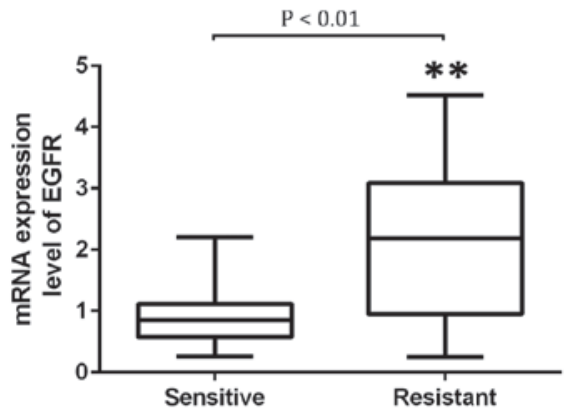

C

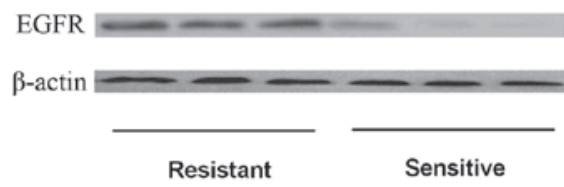

D

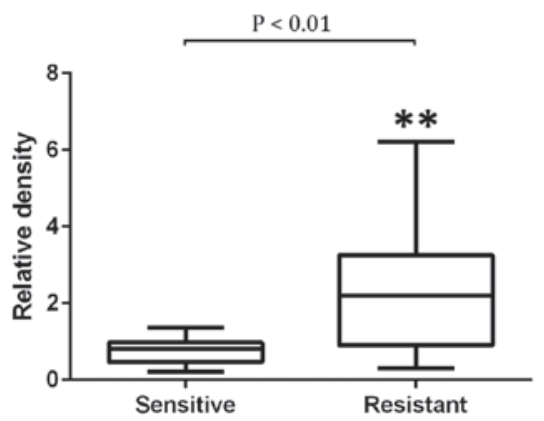

Figure 2. (A) miR-27b was significantly downregulated in NSCLC tissues that were resistant to the treatment of docetaxel compared with those sensitive cases. (B) mRNA and (C and D) protein expression levels of EGFR were significantly upregulated in NSCLC tissues that were resistant to docetaxel when compared with sensitive cases $\left({ }^{* *} \mathrm{P}<0.01\right)$ miR-27b, microRNA-27b; EGFR, epithelial growth factor receptor; NSCLC, non-small cell lung carcinoma.

the regulatory relationship between miR-27b and EGFR, the full length wild-type EGFR 3'-UTR was sub-cloned into psiCHECK2 with a downstream firefly luciferase gene. The two predicted binding sites (200-207 bp and 430-436 bp) were replaced with complementary sequences, labeled as mutant 1 and mutant 2 EGFR 3'-UTR, respectively. Subsequently, A549 cells were transfected with psiCHECK2-EGFR-3'-UTR or psiCHECK2-mutant 1 or mutant 2 EGFR-3'-UTR vectors, together with miR-27b mimics or scramble controls. Following incubation for $48 \mathrm{~h}$, the luciferase activity was examined, and the data revealed that it was significantly reduced in A549 cells co-transfected with wild type or mutant 2 3'-UTR of EGFR 
A

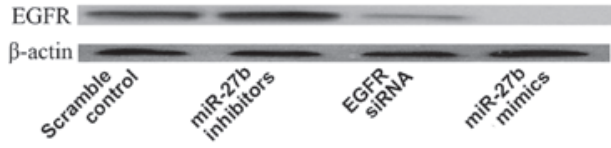

B

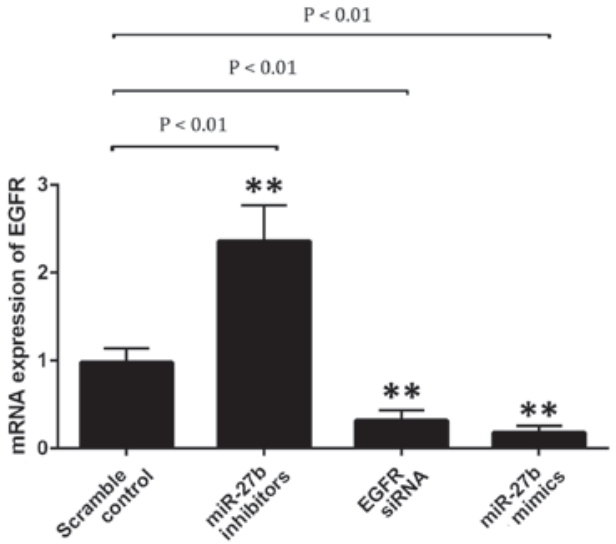

Figure 3. (A) Protein and (B) mRNA expression levels of EGFR in A549 cells transfected with EGFR siRNA or miR-27b mimics were higher in resistant cases compared with the control. ${ }^{* *} \mathrm{P}<0.01$ vs. control miR-27b, microRNA-27b; EGFR, epithelial growth factor receptor siRNA, small interfering RNA.

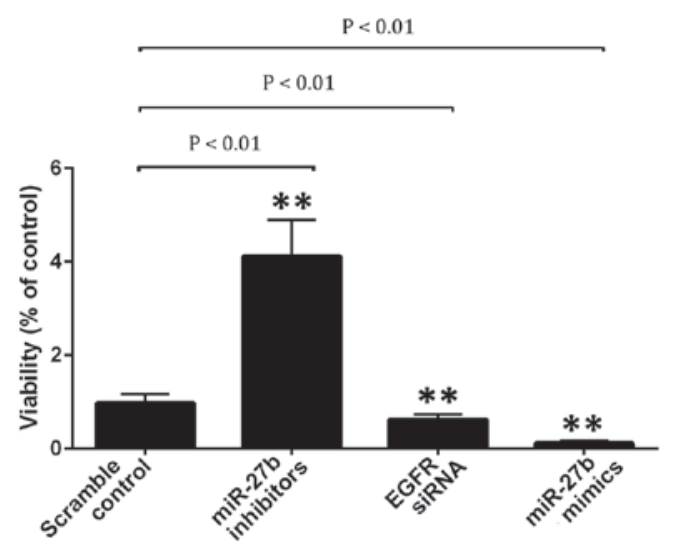

Figure 4. Viability of A549 cells transfected with EGFR siRNA or miR-27b mimics was higher in resistant cases than in the control, while the viability of A549 cells transfected with EGFR siRNA or miR-27b mimics were higher in resistant cases than the control $\left({ }^{* *} \mathrm{P}<0.01 \mathrm{vs}\right.$. control). miR-27b, microRNA-27b; EGFR, epithelial growth factor receptor; siRNA, small interfering RNA.

and miR-27b mimics compared with the scramble control $(\mathrm{P}<0.01$; Fig. 1), suggesting that miR-27b may directly bind to 200-207 bp in the 3-'UTR of EGFR mRNA in A549 cells.

Determination of the expression of miR-27b and EGFR in docetaxel-resistant and-sensitive NSCLC samples. To further verify the regulatory role of miR-27b in EGFR expression, 28 docetaxel-sensitive and 26 docetaxel-resistant NSCLC tissue samples were collected. The expression levels of miR-27b and EGFR were examined. As indicated by Fig. 2A, it was determined that miR-27b was significantly downregulated in NSCLC tissues resistant to the treatment of docetaxel compared with those sensitive to it $(\mathrm{P}<0.01)$. The expression

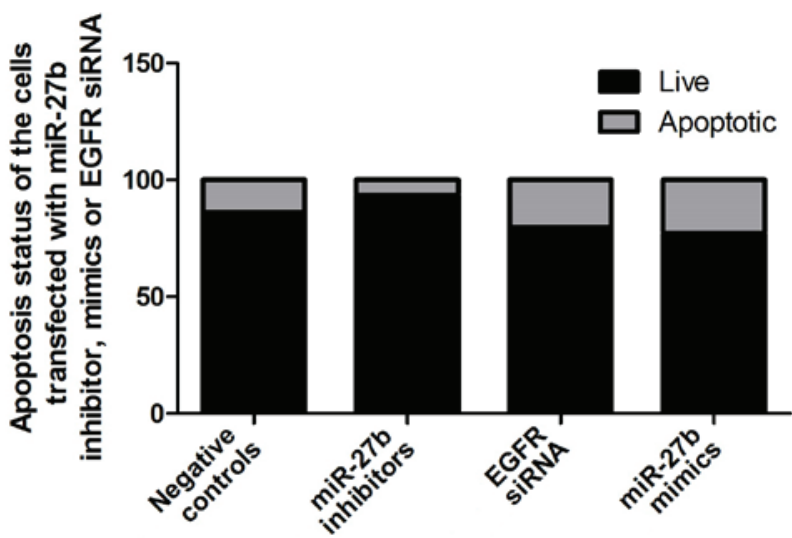

Figure 5. Apoptosis index of A549 cells transfected with scramble, miR-27b inhibitors, EGFR siRNA and miR-27b mimics. miR-27b, microRNA-27b; EGFR, epithelial growth factor receptor; siRNA, small interfering RNA.

of EGFR was determined using semiquantitative RT-PCR and western blot analysis. The mRNA and protein expression of EGFR was significantly upregulated in NSCLC tissues that were resistant to the treatment of docetaxel compared with those sensitive to it $(\mathrm{P}<0.01$; Fig. 2B-D).

miR-27b negatively regulates EGFR expression in A549 cells. A549 cells were transfected with scramble miRNA control, EGFR siRNA, miR-27b mimics or its inhibitor. Subsequently, the protein expression level of EGFR in each group was examined. As indicated in Fig. 3A, the EGFR protein expression level was significantly upregulated following transfection with miR-27b inhibitors $(\mathrm{P}<0.01)$, whilst it was significantly reduced subsequent to transfection with the miR-27b mimics or EGFR siRNA $(\mathrm{P}<0.01)$. To explore the regulatory association between miR-27b and EGFR, the mRNA expression level of EGFR in A549 cells was determined following transfection with the EGFR siRNA, miR-27b mimics or inhibitor. As indicated in Fig. 3B, upregulation of miR-27b and introduction of EGFR siRNA significantly inhibited the protein expression of EGFR in A549 cells $(\mathrm{P}<0.01)$. By contrast, inhibition of miR-27b resulted in a significantly increased protein expression of EGFR in the cells $(\mathrm{P}<0.01)$. Therefore, it was suggested that miR-27b negatively regulates EGFR expression at the transcriptional level in A549 cells.

miR-27b transfection suppresses A546 cell viability by targeting EGFR. To identify the role of miR-27b in the control of the viability of the cells, A549 cells were transfected with EGFR siRNA, and miR-27b mimics or inhibitor. It was determined that the transfection of miR-27b inhibitors significantly increased the viability of the cells by inhibiting apoptosis (Fig. 4). The introduction of EGFR siRNA or miR-27b mimics markedly decreased cell viability and induced the apoptosis of A549 cells (Figs. 4 and 5).

\section{Discussion}

NSCLC is a major health problem worldwide with $>600,000$ cases diagnosed annually in China despite 
improvements in diagnosis and treatment (11). Current treatment options for patients with NSCLC primarily include chemotherapy, surgery, immunotherapy, radiation therapy and targeted therapies, which are primarily dependent on the stage of the disease $(12,13)$. Commercially approved targeted therapies and chemotherapeutic drugs, including docetaxel, cisplatin, carboplatin, paclitaxel, erlotinib, gemcitabine and pemetrexed, are used to treat advanced stage NSCLC (14-18).

Antitubulin agents are important in NSCLC chemotherapy. Taxanes are a family of diterpenes that bind preferentially to microtubules, leading to stabilization and arrest of mitosis (19). As a member of the taxane family, docetaxel is highly active and commonly used as adjuvant therapy following resection of localized tumors in patients with locally advanced NSCLC. However, like the majority of chemotherapeutic agents, cellular resistance limits the clinical success of docetaxel $(20,21)$. It has been determined that miR-27b was significantly downregulated in docetaxel-resistant NSCLC, and may be responsible for the molecular mechanism underlying resistance to docetaxel (9).

In the current study, EGFR was identified as a potential target of miR-27b. The regulatory association between miR-27b and EGFR was validated by a luciferase assay which indicated that the luciferase activity was notably reduced in A549 cells co-transfected with the wild type or mutant 2 3'-UTR of EGFR (430-436 bp was replaced with complementary sequences) and miR-27b mimics, but had minimal effect on those cells transfected with mutant 1 3'-UTR of EGFR (200-207 bp was replaced with complementary sequences). Therefore, miR-27b may directly bind to 200-207 bp in the 3'-UTR of EGFR mRNA in A549 cells. It has been previously determined that miRNAs are involved in regulation of a wide range of biological processes, and inhibition of their function may aid the progression of human malignant tumors (22).

Low levels of miR-27b have been associated with numerous types of human malignancy. A study by Wan et al (23) demonstrated that miR-27b levels were considerably decreased in cell lines and tissue samples of NSCLC, and that excessive expression of miR-27b substantially inhibited NSCLC cell invasion and proliferation. Thereby, suggesting that miR-27b may act as a tumor inhibitor in NSCLC. Previous studies have revealed that miR-27b acts as a tumor suppressor in the development of various malignancies, including prostate cancer, colon cancer and neuroblastoma (24-26). However, other studies have also indicated that miR-27b may promote tumorigenesis in various cancer types. A study by Jin et al (27) demonstrated that miR-27b was significantly upregulated in human breast cancer, and its proliferation was inhibited by knockdown of miR-27b. Another study by Rui et al (9) demonstrated that miR-27b functions as an oncogene in glioma as significant upregulation of miR-27b in glioma cells and tissues was determined. The present study has confirmed the downregulation of miR-27b in docetaxel-resistant NSCLC by measuring and comparing the expression of miR-27b in 28 docetaxel sensitive and 26 docetaxel resistant NSCLC tissue samples. EGFR was validated as a target of miR-27b. Additionally, mRNA and protein expression of EGFR were significantly upregulated in NSCLC tissues that were resistant to docetaxel compared with those that were sensitive.

As a transmembrane growth factor receptor,EGFR exhibits tyrosine kinase activity, which is important for its involvement in controlling the survival and proliferation of cells (28), and it has been indicated that the expression of EGFR was negatively associated with the apoptosis of cancer cells (29). Various mechanisms, including gene mutation, copy number variation, and protein overexpression are associated with the deregulation of cellular activity of EGFR $(30,31)$. Alterations in the quantity or activity of EGFR are not uncommon in lung cancer and are usually associated with numerous pathological features, including poor differentiation, high tumor grading and an unsatisfactory prognosis, suggesting that EGFR may be a therapeutic target for lung cancer (31-34). In the present study, miR-27b negatively regulated the expression of EGFR, as shown by the observation that miR-27b transfection mimics downregulated the expression of EGFR, whereas miR-27b inhibitors upregulated the expression of EGFR. Furthermore, it was demonstrated that transfection with miR-27b mimics significantly suppressed the apoptosis and promoted the viability of A549 cells. By contrast, the introduction of miR-27b inhibitors significantly induced apoptosis and inhibited the proliferation of A549 cells.

In conclusion, EGFR was validated as a target of miR-27b in NSCLC. It also mediated the apoptosis-promoting effect of $\mathrm{miR}-27 \mathrm{~b}$ and the transfection of miR-27 inhibitors significantly promoted the apoptosis of lung cancer cells via targeting EGFR. Therefore, it is possible that EGFR signaling pathway activation underlies the observed downregulation of miR-27b in docetaxel-resistant NSCLC cells, and administration of $\mathrm{miR}-27 \mathrm{~b}$ in a manner that efficiently enhances the level of miR-27b in NSCLC tumor tissue may be a novel method to improve the sensitivity to docetaxel in patients with NSCLC.

\section{References}

1. Jalal SI, Ademuyiwa FO and Hanna NH: The role of maintenance chemotherapy in advanced nonsmall cell lung cancer. Curr Opin Oncol 21: 110-115, 2009.

2. Edelman MJ and Gandara DR: Promising new agents in the treatment of non-small cell lung cancer. Cancer Chemother Pharmacol 37: 385-393, 1996

3. Peng Y, Yu S, Li H, Xiang H, Peng J and Jiang S: MicroRNAs: Emerging roles in adipogenesis and obesity. Cell Signal 26: 1888-1896, 2014

4. Palumbo S, Miracco C, Pirtoli L and Comincini S: Emerging roles of microRNA in modulating cell-death processes in malignant glioma. J Cell Physiol 229: 277-286, 2014.

5. Liu R, Liu X, Zheng Y, Gu J, Xiong S, Jiang P, Jiang X, Huang E, Yang Y, Ge D and Chu Y: MicroRNA-7 sensitizes non-small cell lung cancer cells to paclitaxel. Oncol Lett 8: 2193-2200, 2014.

6. Ning FL, Wang F, Li ML, Yu ZS, Hao YZ and Chen SS: MicroRNA-182 modulates chemosensitivity of human non-small cell lung cancer to cisplatin by targeting PDCD4. Diagn Pathol 9: 143,2014

7. Zhang F, Li Y, Wu H, Qi K, You J, Li X, Zu L, Pan Z, Wang Y, Li Y, et al: MiR-192 confers cisplatin resistance by targeting Bim in lung cancer. Zhongguo fei ai za zhi 17: 384-390, 2014 (In Chinese).

8. Jiang Z, Yin J, Fu W, Mo Y, Pan Y, Dai L, Huang H, Li S and Zhao J: MiRNA 17 family regulates cisplatin-resistant and metastasis by targeting TGFßR2 in NSCLC. PLoS One 9: e94639, 2014.

9. Rui W, Bing F, Hai-Zhu S, Wei D and Long-Bang C: Identification of microRNA profiles in docetaxel-resistant human non-small cell lung carcinoma cells (SPC-A1). J Cell Mol Med 14: 206-214, 2010.

10. Livak KJ and Schmittgen TD: Analysis of relative gene expression data using real-time quantitative PCR and the 2(-Delta Delta C(T)) Method. Methods 25: 402-408, 2001.

11. Walker S: Updates in non-small cell lung cancer. Clin J Oncol Nurs 12: 587-96, 2008. 
12. Gkiozos I, Charpidou A and Syrigos K: Developments in the treatment of non-small cell lung cancer. Anticancer Res 27: 2823-2827, 2007.

13. Bernstein ED, Herbert SM and Hanna NH: Chemotherapy and radiotherapy in the treatment of resectable non-small-cell lung cancer. Ann Surg Oncol 13: 291-301, 2006.

14. Pirker R: Adjuvant chemotherapy in patients with completely resected non-small cell lung cancer. Transl Lung Cancer Res 3 : $305-310,2014$

15. Bi N and Wang L: Superiority of concomitant chemoradiation over sequential chemoradiation in inoperable, locally advanced non-small cell lung cancer: Challenges in the selection of appropriate chemotherapy. Semin Radiat Oncol 25: 122-132, 2015.

16. Matikas A, Mistriotis D, Georgoulias V and Kotsakis A: Current and future approaches in the management of non-small-cell lung cancer patients with resistance to EGFR TKIs. Clin Lung Cancer 16: 252-261, 2015

17. Pilkington G, Boland A, Brown T, Oyee J, Bagust A and Dickson R: A systematic review of the clinical effectiveness of first-line chemotherapy for adult patients with locally advanced or metastatic non-small cell lung cancer. Thorax: Feb 6, 2015 (Epub ahead of print)

18. Paul I and Jones JM: Apoptosis block as a barrier to effective therapy in non small cell lung cancer. World J Clin Oncol 5: 588-594, 2014.

19. Horwitz SB: Taxol (paclitaxel): Mechanisms of action. Ann Oncol 5 (Suppl 6): S3-S6, 1994.

20. Geney R, Ungureanu M, Li D and Ojima I: Overcoming multidrug resistance in taxane chemotherapy. Clin Chem Lab Med 40: 918-925, 2002.

21. McGrogan BT, Gilmartin B, Carney DN and McCann A: Taxanes, microtubules and chemoresistant breast cancer. Biochim Biophys Acta 1785: 96-132, 2007.

22. Tay FC, Lim JK, Zhu H, Hin LC and Wang S: Using artificial microRNA sponges to achieve microRNA loss-of-function in cancer cells. Adv Drug Deliv Rev 81: 117-127, 2015.

23. Wan L, Zhang L, Fan K and Wang J: MiR-27b targets LIMK1 to inhibit growth and invasion of NSCLC cells. Mol Cell Biochem 390: 85-91, 2014.
24. Ye J, Wu X, Wu D, Wu P, Ni C, Zhang Z, Chen Z, Qiu F, Xu J and Huang J: miRNA-27b targets vascular endothelial growth factor $\mathrm{C}$ to inhibit tumor progression and angiogenesis in colorectal cancer. PLoS One 8: e60687, 2013.

25. Ishteiwy RA, Ward TM, Dykxhoorn DM and Burnstein KL: The microRNA-23b/-27b cluster suppresses the metastatic phenotype of castration-resistant prostate cancer cells. PLoS One 7: e52106, 2012.

26. Lee JJ, Drakaki A, Iliopoulos D and Struhl K: MiR-27b targets PPAR $\gamma$ to inhibit growth, tumor progression and the inflammatory response in neuroblastoma cells. Oncogene 31 : 3818-3825, 2012.

27. Jin L, Wessely O, Marcusson EG, Ivan C, Calin GA and Alahari SK: Prooncogenic factors miR-23b and miR-27b are regulated by Her $2 / \mathrm{Neu}, \mathrm{EGF}$, and TNF- $\alpha$ in breast cancer. Cancer Res 73: 2884-2896, 2013.

28. Jemal A, Siegel R, Xu J and Ward E: Cancer statistics, 2010. CA Cancer J Clin 60: 277-300, 2010.

29. Gu J, Ding JY, Lu CL, Lin ZW, Chu YW, Zhao GY, Guo J and Ge D: Overexpression of CD88 predicts poor prognosis in non-small-cell lung cancer. Lung Cancer 81: 259-265, 2013.

30. Edelman MJ: Novel taxane formulations and microtubule-binding agents in non-small-cell lung cancer. Clin Lung Cancer 10: S30-S34, 2009.

31. Morales-Cano D, Calviño E, Rubio V, Herráez A, Sancho P Tejedor MC and Diez JC: Apoptosis induced by paclitaxel via Bcl-2, Bax and caspases 3 and 9 activation in NB4 human leukaemia cells is not modulated by ERK inhibition. Exp Toxicol Pathol 65: 1101-1108, 2013.

32. Kavallaris M: Microtubules and resistance to tubulin-binding agents. Nat Rev Cancer 10: 194-204, 2010.

33. Wan YF, Guo XQ, Wang ZH, Ying K and Yao MH: Effects of paclitaxel on proliferation and apoptosis in human acute myeloid leukemia HL-60 cells. Acta Pharmacol Sin 25: 378-384, 2004.

34. Xu R, Sato N, Yanai K, Akiyoshi T, Nagai S, Wada J, Koga K, Mibu R, Nakamura M and Katano M: Enhancement of paclitaxel-induced apoptosis by inhibition of mitogen-activated protein kinase pathway in colon cancer cells. Anticancer Res 29: 261-270, 2009. 